\title{
Sur les courbes qui engendrent par translation une surface minima.
}

\author{
par René Lagrange (à Dijon, France) \\ A M. Enrico Bompiani pour son Jubile scientifique
}

\begin{abstract}
Résumé. - Les courbes gauches qui engendrent par translation une surface minima sont les courbes à cône directeur quadratique pour lesquelles $T R^{z}$ const. On donne leurs équations intrinséques et leur représentation explicite. On détermine aussi les courbes planes qui ont la même propriété.
\end{abstract}

Si une courbe $C$ engendre par translation une surface minima $S$, $S$ est doublement de translation. Des génératrices par translation de $S$ sont $C$, la directrice $C_{1}$ associée à $C$, et les courbes de longueur nulle. Un théorème de Lophus lie entraine que la rénnion des cônes directenrs de ces directrices est un còne algébrique du quatrième ordre. Le còne directeur commun aux lignes de longueur nulle étant le cône isotrope, la réunion des cônes directeurs de $C$ et $C_{1}$ est un cône quadratique.

Nous nous proposons de détérminer les génératrices telles que $C$. Le còne directeur est de degré au plus égal à 2 . Si c'est une droite, $C$ est elle-même une droite; $S$ est un cylindre, et les surfaces minima cylindriques sont les plans. Cette réponse banale étant écartée, les seules courbes à examiner sont les courbes planes et celles à cône directeur quadratique. Dans le premier cas, $C$ et $C_{1}$ sont planes, qu' on suppose naturellement dans des plans sécants. Dans le second cas, $C$ et $C_{1}$ ont le même cône directeur, donc la même indicatrice sphérique, qui est une conique sphérique.

Avant de poursuivre. nous établissons une expression de la courbure géodésique d' une telle conique.

1. Nous définissons cette conique $\gamma$ par l'intersection de la sphère unitaire $\Sigma$ d'équation

$$
x_{1}^{2}+x_{2}^{2}+x_{3}^{2}-1=0,
$$

en axes rectangulaires $0 x_{1} x_{2} x_{3}$, et $\mathrm{du}$ cône $\Gamma$ d'équation

$$
\sum_{i=1}^{3} \frac{x_{i}^{2}}{a_{i}}=0
$$


Dans le cas général où nous nous plaçons, $\Gamma$ n'est pas de révolution. Sa réalité suppose $a_{1} a_{2} a_{3}<0$, et, sans restreindre la généralité, on peut faire $a_{1}>a_{2}>0>a_{8}$.

Une représentation paramétrique de $\Gamma$, avec les deux paramétres $q$ et $H$, est

$$
\frac{x_{i}^{2}}{a_{i}}=\frac{\left(q+a_{i}\right)\left(a_{j}-a_{k}\right)}{H}, \quad i=1,2,3
$$

où $i, j, k$ désigne une permutation circulaire de $1,2,3$. Sur $\gamma, q$ et $H$ sont liés par l'équation de $\Sigma$, ce qui donne tout de suite

$$
H=\sum_{i=1}^{3} a_{i}{ }^{2}\left(a_{j}-a_{k}\right)=-\left|\begin{array}{ccc}
1 & 1 & 1 \\
a_{1} & a_{2} & a_{3} \\
a_{1}^{2} & a_{2}^{2} & a_{3}^{2}
\end{array}\right|=-\left(a_{1}-a_{2}\right)\left(a_{2}-a_{3}\right)\left(a_{8}-a_{1}\right)
$$

Ainsi $H$ est une constante, positive avec nos hypothèses. Le réalité des $x_{i}$ réduit le domaine de variation de $q$ au segment $\left[-a_{1},-a_{2}\right]$.

De la différentiation de (1)

$$
\frac{2}{x_{i}} d x_{i}=\frac{d q}{q+a_{i}}
$$

résulte, pour le $d s^{2}$ de $\gamma$, l'expression

$$
\left(\frac{d s}{d q}\right)^{2}=\frac{1}{4} \sum_{i=1}^{3} \frac{x_{i}{ }^{2}}{\left(q+a_{i}\right)^{2}}=\frac{\sum_{i=1}^{3} a_{i}\left(a_{j}-a_{k}\right)\left(q+a_{j}\right)\left(q+a_{k}\right)}{4 H\left(q+a_{1}\right)\left(q+a_{2}\right)\left(q+a_{3}\right)} .
$$

On voit tout de suite que le numératenr se réduit a

$$
q \sum_{i=1}^{3} a_{i}\left(a_{j}^{2}-a_{k}^{2}\right)=-H q
$$

done

$$
\left(\frac{d s}{d q}\right)^{2}=\frac{-q}{4\left(q+a_{1}\right)\left(q+a_{2}\right)\left(q+a_{3}\right)}
$$

quotient de deux termes positifs. En posant

$$
\frac{d u}{d q}=\frac{1}{2 \sqrt{\left(q+a_{1}\right)\left(q+a_{2}\right)\left(q+a_{s}\right)}}
$$


$q$ est une fonction elliptique de $u$, et l'on peut choisir la détermination

$$
d s=\sqrt{-q} d u \text {. }
$$

En posant $\frac{d q}{d u}=q^{\prime}$, les cosinus directeurs de la tangente $\vec{t}$ de $\gamma$ sont

$$
\lambda_{i}=\frac{d x_{i}}{d s}=\frac{x_{i}}{2\left(q+a_{i}\right)} \frac{q^{\prime}}{\sqrt{-q}}=\frac{q^{\prime}}{2 H \sqrt{-q}} \frac{a_{i}\left(a_{j}-a_{k}\right)}{x_{i}} .
$$

Un vecteur unitaire normal à $\Sigma$ est le vecteur $\vec{v}$ de composantes $x_{1}, x_{2}, x_{3}$ : done la normale géodésique $\vec{g}=\vec{v} \wedge \vec{t}$ de $\gamma$ a les cosinus directeurs

$$
\mu_{i}=x_{j} \lambda_{k}-x_{k} \lambda_{j}=\frac{q^{\prime}}{2 H \vee \overline{-q}} \frac{a_{k}\left(a_{i}-a_{j}\right) x_{j}{ }^{2}-a_{j}\left(a_{k}-a_{i}\right) x_{k}{ }^{2}}{x_{j} x_{k}} .
$$

Grâce à (1) et (2), le numérateur de cette dernière fraction s' écrit

$$
\frac{1}{H} a_{j} a_{k}\left(a_{i}-a_{j}\right)\left(a_{j}-a_{k}\right)\left(a_{k}-a_{i}\right)=-a_{j} a_{k},
$$

et

$$
\mu_{i}=-\frac{q^{\prime}}{2 H V-q} \frac{a_{j} a_{k}}{x_{j} x_{k}}
$$

D'après

$$
\frac{1}{\bar{\lambda}_{i}} \frac{d{\grave{\Lambda_{i}}}_{d s}}{d s}=-\frac{1}{x_{i}} \frac{d x_{i}}{d s}+\frac{V \overline{-q}}{q^{\prime}} \frac{d}{d s}\left(\frac{q^{\prime}}{\sqrt{-q}}\right),
$$

la courbure géodésique de $\gamma$ est

$$
\overline{R_{g}}=\sum_{i=1}^{3} \mu_{i} \frac{d \lambda_{1}}{d s}=-\sum_{i=1}^{s} \frac{\lambda_{i}{ }^{2} \mu_{i}}{x_{i}}=\frac{q^{\prime}}{2 H \sqrt{-q}} \frac{1}{x_{1} x_{2} x_{3}} \underset{i=1}{\sum_{i=1}^{3}} \lambda_{i}{ }^{2} a_{j} a_{k}
$$

Or

$$
\lambda_{i}{ }^{2} a_{j} a_{k}=-\frac{q^{\prime 2} a_{1} a_{2} a_{3}}{4 H q} \frac{a_{i}\left(a_{j}-a_{k}\right)^{2}}{H x_{i}{ }^{2}}=-\frac{q^{2} a_{1} a_{2} a_{3}}{4 H q} \frac{a_{j}-a_{k}}{q+a_{i}},
$$

et l'on est conduit à former

$$
\underbrace{3}_{i=1} \frac{a_{j}-a_{k}}{q+a_{i}}=\frac{\stackrel{3}{i=1}_{i=1}\left(a_{j}-a_{k}\right)\left(q+a_{j}\right)\left(q+a_{k}\right)}{\left(q+a_{1}\right)\left(q+a_{2}\right)\left(q+a_{3}\right)}=\frac{\sum_{i=1}^{s} a_{j} a_{k}\left(a_{j}-a_{k}\right)}{\left(q+a_{1}\right)\left(q+a_{2}\right)\left(q+a_{3}\right)}=\frac{4 H}{q^{\prime 2}} .
$$


Il vient ainsi

$$
\frac{1}{R_{g}}=\frac{q^{\prime}}{2 H(-q)^{\frac{3}{2}}} \frac{a_{1} a_{2} a_{3}}{x_{1} x_{2} x_{3}}
$$

Son carré

$$
\frac{1}{R_{g}^{2}}=\frac{q^{2} a_{1} a_{2} a_{s}}{-4 H^{2} q^{3}} \prod_{i=1}^{3} \frac{a_{i}}{x_{i}^{2}}
$$

prend, grace à (1) et (5), la forme remarquable

$$
\frac{1}{R_{g}^{2}}=\frac{a_{1} a_{2} a_{s}}{q^{3}}
$$

Les petits cercles de $\Sigma$ sont fournis par le cas limite où $a_{2}$ tend vers $a_{1}$. Les expressions (1) de $x_{1}^{2}$ et $x_{2}^{2}$ sont alors indéterminées, avec une vraie valeur arbitraire de $\frac{q+a_{1}}{a_{1}-a_{2}}$, lorsque $q$ tend egalement vers $a_{1} .(10)$ est encore vala. ble, aree la valeur constante $\frac{-a_{3}}{a_{1}}$. Les grands cercles correspondent au cas limite $a_{1}>a_{2}=0>a_{3} ; \frac{1}{R_{g}}$ est bien nul, et réciproquement.

Remarque. - Le paramètre $q$ de la représentation de $\gamma$ est, à un facteur près, la puissance $\frac{2}{3}$ du rayon de courbure géodésique. Avec une conique plane

$$
\frac{x_{1}^{2}}{a_{1}}+\frac{x_{2}^{2}}{a_{2}}-1=0, \quad a_{1}>a_{2}
$$

la représentation

$$
\frac{x_{1}^{2}}{a_{1}}=\frac{q+a_{1}}{2 e}, \frac{x_{2}^{2}}{a_{2}}=\frac{q+a_{2}}{-2 e}
$$

où $e=\frac{a_{1}-}{2} \underline{a_{2}}$, utilise de même un paramètre $q$ tel que la courbure $\frac{1}{\tilde{I}}$ vérifie la formule

$$
\frac{1}{R^{2}}=-\frac{a_{1} a_{2}}{q^{3}}
$$

analogue à (10). 
2. Les courbes $C$ de l'espace qui ont un cône directeur quadratique sont celles dont l'indicatrice sphérique est une courbe $\gamma$ du type qui précède. Étant donnó les relations de Serret-Frenet de $C$,

$$
\left\{\begin{array}{l}
d A=\vec{t} d s, \\
d \vec{t}=\vec{n} d \sigma \\
d \vec{n}=-\vec{t} d \sigma+\vec{b} d \tau, \\
d \vec{b}=-\vec{n} d \tau,
\end{array}\right.
$$

avec la courbure $\frac{1}{R}=\frac{d \sigma}{d s}$, et $\frac{1}{T}=\frac{d \tau}{d s}$, l'elément d'arc de $\gamma$ est $d \sigma$, et sa courbure géodésique $\frac{1}{R_{g}}=\frac{R}{T}$. Les courbes $C$ en question sont donc celles qui admettent des équations intrinsèques du type

$$
\left\{\begin{array}{l}
d \sigma=\sqrt{-q} d u, \\
\left(\frac{T}{R}\right)^{2}=\frac{q^{3}}{a_{1} a_{2} a_{3}}
\end{array},\right.
$$

où $q$ est la fonetion elliptique de $u$ definie par exemple par

$$
u=\frac{1}{2} \int_{-a_{1}}^{q} \frac{d q}{\sqrt{\left(q+a_{1}\right)\left(q+a_{2}\right)\left(q+a_{3}\right)}}
$$

avec $-a_{1} \leq q \leq-a_{2}<0<-a_{3} . u$ décrit un segment $\left[0, u_{0}\right]$ lorsque $q$ dé. crit $\left[-a_{1},-a_{2}\right]$. $C$ n'est complétement définie que par l'adjonction à (12) d'une equation $\frac{d s}{d u}=f(u)$.

Remarques. - Les $a_{i}$ ne sont définis qu'à un facteur positif près $\lambda$. En remplaçant de même $q$ par $\lambda q, u$ est divisé par $\sqrt{\lambda}$.

Bien entendu, $q(u)$ s'exprime par la fonction de WeInRstrass en posant

$$
\sum_{i=1}^{s} a_{i}=3 h, \quad a_{i}=h-e_{i}, \quad q=p-h,
$$

mais e' est inutile ici.

3. Appliquons maintenant ces préliminaires à la recherche des surfaces minima $S$ engendrées par la translation d'une courbe réelle. $C$. Soit $C_{1}$ une directrice associée, qui joue le mème rôle que $C$. Nous supposons que leurs courbures ne sont pas identiquement nulles. Soit $A(u)$ et $B(v)$ leurs points 
courants, vérifiant dans le cas général des équations intrinsèques du type (12), avec les mèmes $a_{i}$. Lorsque $C$ et $C_{1}$ sont planes, dans des plans sécants, on peut prendre $0 x_{1}$ parallèle à cette intersection, ce qui revient à faire $a_{2}=a_{3}=0$ dans les formules générales.

Avec des notations dont la signification est évidente, écrivons ces rela. tions (12) pour $C$ et $C_{1}$, soit

$$
\left\{\begin{array}{l}
d \sigma_{u}=\sqrt{-q(u)} \mathrm{d} u . \\
\frac{d \sigma_{u}}{d \tau_{u}}=\frac{[-q(u)]^{\frac{3}{2}}}{\sqrt{-a_{1} a_{2} a_{3}}} . \\
d \sigma_{v}=\sqrt{-q(v)} d v, \\
\frac{d \sigma_{v}}{d \tau_{v}}=\frac{[-q(v)]^{\frac{3}{2}}}{\sqrt{-a_{1} a_{2} a_{3}}} .
\end{array}\right.
$$

$C$ et $C_{1}$ sont précisées par la donnée supplémentaire des fonctions $\frac{d s_{u}}{d u}$ et $\frac{d s_{v}}{d v}$. Les radicaux ont la même signification arithmétique dans $(14)$ et $\left(14^{\prime}\right)$ puisque, sur l' indicatrice commune $\gamma, \frac{1}{K_{g}}=\frac{R}{T}$ est une fonction continue qui ne devient ni nulle ni infinie dans l'intervalle de variation $\left[-a_{1},-a_{2}\right]$ de $q$.

Le point courant $M$ de la surface $S$ est

$$
M(u, v)=A(u)+B(v)
$$

de sorte que les tangentes du réseau de référence sont

$$
\frac{\partial M}{\partial u}=\frac{d A}{d u}=\vec{t}_{u} \frac{d s_{u}}{d u}, \quad \frac{\partial M}{\partial v}=\frac{d B}{d v}=\vec{t}_{v} \frac{d s_{v}}{d v} ;
$$

les coefficients $E, G$ de la première forme de GaUss sont

$$
E=\left(\frac{d s_{u}}{d u}\right)^{2}, \quad G=\left(\frac{d s_{v}}{d v}\right)^{2}
$$

Il vient ensuite

$$
\frac{\partial^{2} M}{\partial u^{2}}=\overrightarrow{t_{u}} \frac{d^{2} s_{u}}{d u^{2}}+\overrightarrow{n_{u}} \frac{d \sigma_{u}}{d u} \frac{d s_{u}}{d u}
$$

donc les coefficients $D, D^{\prime \prime}$ de la deuxième forme de Gauss sont

$$
D=-\left(\frac{d s_{u}}{d u}\right)^{2} \frac{d s_{v}}{d v} \frac{d \sigma_{u}}{d u}\left(\overrightarrow{b_{u}} \overrightarrow{t_{v}}\right), \quad D^{\prime}=\frac{d s_{u}}{d u}\left(\frac{d s_{v}}{d v}\right)^{2} \frac{d \sigma_{v}}{d v}\left(\overrightarrow{b_{v}} \overrightarrow{t_{u}}\right)
$$


$S$ est minima pourvu que $D G+D^{\prime \prime} E=0$, donc, après division $\operatorname{par}\left(\frac{d s_{u}}{d u}\right)^{2}\left(\frac{d s_{v}}{d v}\right)^{2}$, pourvu que

$$
\frac{d s_{v}}{d v} \frac{d \sigma_{u}}{d u}\left(\overrightarrow{b_{u}} \overrightarrow{t_{v}}\right)-\frac{d s_{u}}{d u} \frac{d \sigma_{v}}{d v}\left(\overrightarrow{b_{v}} \overrightarrow{t_{u}}\right)=0
$$

Cette condition est tout à fait générale. Lorsque $C$ est gauche, il en est de mème pour $C_{1}$, et on peut adjondre les systèmes (14), $\left(14^{\prime}\right)$ relatifs à l'in. dicatrice commune $\gamma$. Avec les notations du paragraphe 1 , il vient

$$
\begin{aligned}
\overrightarrow{b_{u}} \overrightarrow{t_{v}} & =\overrightarrow{g_{u}} \overrightarrow{v_{v}}=\sum_{i=1}^{3} \mu_{i}(u) x_{i}(v)=\frac{-q^{\prime}(u)}{2 H \sqrt{-q(u)}} \sum_{i=1}^{3} \frac{a_{j} a_{k} x_{i}(v)}{x_{j}(u) x_{k}(u)} \\
& =\frac{-a_{1} a_{2} a_{3} q^{\prime}(u)}{2 H V-q(u) x_{3}(u) x_{2}(u) x_{3}(u)} \sum_{i=1}^{3} \frac{x_{i}(u) x_{i}(v)}{a_{i}}
\end{aligned}
$$

La somme est symétrique en $u, v$; d'après $(9)$, son facteur n' est rien d'autre que

$$
\frac{q(u)}{R_{g}(u)}=q(u) \frac{d \tau_{u}}{d \sigma_{u}}=-\frac{\sqrt{-a_{1} a_{2} a_{s}}}{\sqrt{-q(u)}}
$$

et (16) se réduit à

$$
\frac{d s_{v}}{d v}=\frac{d s_{u}}{d u} .
$$

Le valeur commune de ces deux membres est constante, et fournit la donnée qui permet de préciser la nature de $C$ et $C_{1}$. Ces courbes, supposées gauches, sont lióes par une translation. Le facteur constant arbitraire positif $\lambda$ dont on dispose pour définir les $a_{i}$, et le sens de la mesure de $s$ permet de donner à (I7) la valeur 1 , de sorte que $C$ et $C_{1}$ soient définies par des équations intrinsèques

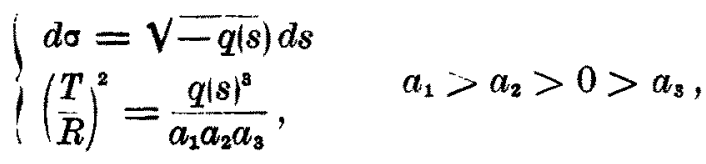

avec, par exemple,

$$
s=\frac{1}{2} \int_{-a_{1}}^{q} \frac{d q}{\sqrt{\left(q+a_{1}\right)\left(q+a_{2}\right)\left(q+a_{8}\right)}} .
$$


Dans ces formules, on ne peut plus modifier les $a_{i}$; la multiplication des $a_{i}$ et de $q$ par un facteur $\lambda>0$ conserve (18) a condition de multiplier $s$ par $\frac{1}{\sqrt{\lambda}} ; C$ subit l'homothétie de rapport $\frac{1}{\sqrt{\lambda}} \cdot(18)$ s'ecrit encore $^{\prime}$

$$
\left\{\begin{array}{l}
1=\sqrt{-q(s)} \\
\frac{1}{T R^{2}}= \pm \sqrt{-a_{1} a_{2} a_{3}}=\text { const. }
\end{array}\right.
$$

Les courbes réelles gauches dont la translation engendre une surface minima sont les courbes à cône directeur quadratique pour lesquelles $T R^{2}=$ const..

Elles dépendent. en grandeur, des trois paramètres $a_{i}$, qui n'interviennent que par leurs rapports dans la définition du cône. Les surfaces minima obtenues sont également les lieux des milieux des cordes d'une courbe du type (20), ce que H. Poincaré a appelé "surfaces de translation distinguées».

Dans le cas limite $a_{1}=a_{2}>0>a_{3}, C$ est une hélice circulaire, puisque $q=$ const., et $S$ est le lieu des milieux des cordes d'une telle hélice.

4. Lorsque $C$ et $C_{1}$ sont planes, nous pouvons faire $d s_{u}=d u, d s_{v}=d v$, et choisir des axes cartésiens rectangulaires $0 x, 0 y, 0 z$, où $0 x$ est l'intersection des plans de ces deux courbes. Prenons le plan de $C$ ponr plan 0xy. Si $\overrightarrow{i,} \vec{j}, \vec{k}$ désignent les vecteurs unitaires des trois axes, le plan $0 x Y$ de $C_{1}$ est tel que $0 Y$ soit parallèle an vecteur $\vec{j} \cos \theta+\vec{k} \sin \theta$. Si

$$
x=x(u), \quad y=y(u), \quad z=0,
$$

représentent $C$, tandis que $C_{1}$ a des équations

$$
x=X(v), \quad y=Y(v) \cos \theta, \quad z=Y(v) \sin \theta,
$$

les éléments de l'équation générale (16) sont ici

$$
\begin{gathered}
\overrightarrow{b_{u}}=\vec{k}, \quad \overrightarrow{t_{u}}=\frac{d x}{d u} \vec{i}+\frac{d y}{d u} \vec{j}, \\
\overrightarrow{b_{v}}=-\vec{j} \sin \theta+\vec{k} \cos \theta, \quad \overrightarrow{t_{v}}=\frac{d X}{d v} \vec{i}+\frac{d Y}{d v}(\vec{j} \cos \theta+\vec{k} \sin \theta) .
\end{gathered}
$$

Par suite

$$
\overrightarrow{b_{u}} \overrightarrow{t_{v}}=\frac{d Y}{d v} \sin \theta, \quad \overrightarrow{b_{v}} \overrightarrow{t_{u}}=-\frac{d y}{d u} \sin \theta, \quad \sin \theta \neq 0,
$$


et (16) s' écrit

$$
\frac{d \sigma_{u}}{d u} \frac{d Y}{d v}+\frac{d \sigma_{v}}{d v} \frac{d y}{d u}=0
$$

soit

$$
\frac{d y(u)}{d \sigma_{u}}=-\frac{d Y}{d \sigma_{v}}=\mathrm{const}=a .
$$

A une translation près, l'équation

$$
\frac{d y}{d \sigma}=a
$$

donne tout de suite

$$
\frac{d y}{d x}=\operatorname{tg} \sigma=\operatorname{tg} \frac{y}{a}
$$

d'où l'équation de $C$

$$
x=a \log \left|\sin \frac{y}{a}\right|
$$

$C$ a une infinité de branches, se déduisant de l'une d'elles par translation parallèle à $0 y$, de mesure naj (n entier). Par exemple, une branche est fournie par

$$
\sin \frac{y}{a}=e^{\frac{x}{a}},-\infty<x<0,0<y<a \pi, a>0 .
$$

L'équation de $C_{1}$, également à une translation près, est

$$
x=-a \log \left|\sin \frac{Y}{a}\right| \text {. }
$$

Ainsi, $C$ et $C_{1}$ sont encore égales, mais, en tant que directrice et génératrice associés, elles ne sont pas liées par translation. $C_{1}$ se déduit de $C$ par une symétrie par rapport à la base $0 y$ de $C$, suivie d'une rotation quelconque autour d'une asymptote.

On retrouve (22) en résolvant (18) dans le cas limite $a_{2}=a_{3}=0$, en posant $a_{1}=\frac{1}{a^{2}} ;$ puisque $-\frac{1}{a^{2}} \leq q \leq 0$, on pent faire

$$
q=\frac{-1}{a^{2} \operatorname{ch}^{2} t}
$$


(19) se réduit à $s=a t$, et la première équation (18) devient

$$
R=a \operatorname{ch} \frac{s}{a}
$$

Ceci s'écrit encore

$$
d \sigma=\frac{d s}{a \operatorname{ch} t}=\frac{d t}{\operatorname{ch} t}
$$

ce qui permet de faire $e^{t}=\operatorname{tg} \frac{\sigma}{2}, R=\frac{a}{\sin \sigma}$, avee $0<\sigma<\pi$. Enfin, à une translation près,

$$
\begin{aligned}
& x=\int \cos \sigma d s=a \int \operatorname{cotg} \sigma d \sigma=a \log \mid \sin \sigma, \\
& y=\int \sin \sigma d s=a \sigma,
\end{aligned}
$$

c'est-à-dire (22). Cependant, cette méthode ne fournit pas le lien géométrique entre $C$ et $C_{1}$.

5. Equations explicites de la génératrice générale $C$. Bien que $q$ soit une fonction elliptique de l'are $s$, les expressions des coordonnées $x_{i}$ de $C$ en fonction de $q$ relèvent d'intégrales éémentaires. Pour suivre le point courant $M$ de $C$ lorsque $q$ oscille dans le segment $\left[-a_{1},-a_{2}\right]$, précisons le déplace. ment du point courant $m$ de l'indicatrice sphérique $\gamma$. Celle-ci se décompose en deax courbes symetriques l' une de l'autre par rapport à l'origine, et il suffit de n'en considérer qu' une. Celle-ci se compose de quatre arcs $\widehat{m}_{0} m_{1}, \widehat{m_{1} m_{2}}, \widehat{m_{2} m_{3}}, \widehat{m_{3} m_{0}}$, limités aux plans de cordonnées $x_{1}=0, x_{2}=0$, et orthogonaux à ces plans à leurs extrémités; deux ares contigus sont symétriques l'un de l'autre par rapport au plan qui les sépare; chacun d'eux est décrit par $m$ lorsque $q$ décrit $\left[-a_{1},-a_{2}\right]$ dans un sens ou dans l'autre. En désignant par $\xi_{i}(q)$ les coordonnées du point courant $m$ de $\widehat{m_{0} m_{1}}$, parcouru lorsque $q$ croit de $-a_{1} \grave{a}-a_{2}$, on peut prendre les racines carrées de (1) sous la forme

$$
\xi_{i}(q)=\sqrt{\frac{a_{i}\left(a_{j}-a_{k}\right)\left(q+a_{i}\right)}{H}} \quad-a_{1} \leq q \leq-a_{2},
$$

et $\left.\xi_{2} \mid-a_{2}\right)=0$. L'arc suivant ${\widehat{m_{1} m_{2}}}_{2}$ est le symétrique de $\widehat{m}_{0} \boldsymbol{m}_{1}$ par rapport à $x_{12}=0$, et son point courant a les coordonnees

$$
\bar{\xi}_{1}(q)=\xi_{1}(q), \quad \bar{\xi}_{2}(q)=-\xi_{2}(q), \quad \bar{\xi}_{3}(q)=\xi_{8}(q), \quad-a_{2} \geq q \geq-a_{1},
$$


avec $\xi_{1}\left(-a_{1}\right)=0$. L'arc suivant ${\widehat{m_{2} m_{3}}}_{3}$ est symétrique de $\widehat{m}_{1} m_{2}$ par rapport à $x_{1}=0$, et son point courant a les coordonnées

$$
\overline{\overline{\xi_{1}}}(q)=-\xi_{1}(q), \overline{\bar{\xi}_{2}}(q)=-\xi_{2}(q), \overline{\xi_{3}}(q)=\xi_{3}(q),-a_{1} \leq q \leq-a_{2}
$$

cet arc est symétrique de $\widetilde{m}_{0} m_{1}$ par rapport à l'axe $0 x_{3} ; 1$ arc suivant ${\widehat{m_{3} m_{0}}}$

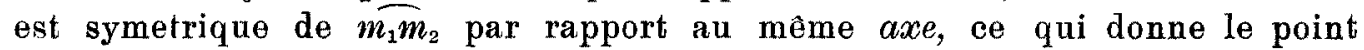
courant

$$
\overline{\bar{\xi}}_{1}(q)=-\xi_{1}(q), \overline{\overline{\vec{\xi}}}_{2}(q)=\xi_{2}(q), \overline{\overline{\bar{\xi}}}_{3}(q)=\xi_{3}(q), \quad-a_{2} \geq q \geq-a_{1} .
$$

6. Lorsque $m$ décrit $\gamma$ dans l'ordre $m_{0} m_{1} m_{2} m_{3} m_{0}$, le point associé $M$ de $C$ décrit un arc $M_{0} M_{1} M_{2} M_{3} M_{4}$. Si l'on place $M_{0}$ à l'origine, et si on observe que $q$ croit pour l'arc $\widehat{M_{0} M_{1}}$, les coordonnés $x_{i}(q)$ du point courant $M$ de cet arc sont

$$
x_{i}(q)=\int_{-a_{1}}^{q} \xi_{i}(q) \frac{d s}{d q} d q=\int_{-a_{1}}^{q} \xi_{i}(q)\left|\frac{d s}{d q}\right| d q
$$

Compte tenu de (19) et (24), ceci se réduit à

$$
x_{i}(q)=\frac{1}{2} \int_{-a_{3}}^{q} \sqrt{\frac{a_{i}\left(a_{j}-a_{k}\right)}{H\left(q+a_{j}\right)\left(q+a_{k}\right)}} d q
$$

Ainsi,

$$
x_{3}(q)=\sqrt{\frac{-a_{3}\left(a_{1}-a_{2}\right)}{H}} \int_{-a_{1}}^{q} \frac{d q}{2 \sqrt{\left(q+a_{1}\right)(}=\overline{\left.-q-a_{2}\right)}} .
$$

En posant

$$
\sqrt{-q-a_{2}}=\sqrt{a_{1}-a_{2}} \cos \varphi, \quad \sqrt{q+a_{1}}=\sqrt{a_{1}-a_{2}} \sin \varphi,
$$

de sorte que $\varphi$ croisse de 0 à $\frac{\pi}{2}$ lorsque $q$ croit de $-a_{1}$ à $-a_{2}$, on a

$$
x_{3}(q)=\sqrt{-\frac{-a_{3}\left(a_{1}-a_{2}\right)}{H}} \varphi .
$$

On a de même

$$
x_{2}(q)=\sqrt{\frac{a_{2}\left(a_{1}-a_{3}\right)}{H}} \int_{-a_{1}}^{q} 2 \backslash\left(q+a_{1}\right)\left(-\frac{d q}{\left.q-a_{3}\right)}=\sqrt{\frac{a_{2}\left(a_{1}-a_{3}\right)}{H}} \psi,\right.
$$


en posant

$$
\sqrt{-q-a_{3}}=\sqrt{a_{1}-a_{3}} \cos \psi, \sqrt{q+a_{1}}=\sqrt{a_{1}-a_{3}} \sin \psi
$$

de sorte que $\psi=\operatorname{Arcsin}\left(\sqrt{\frac{a_{1}-a_{2}}{a_{1}-a_{3}}} \sin \varphi\right)$ croit de 0 a Arc sin $\sqrt{\frac{a_{1}-a_{2}}{a_{1}-a_{3}}}$. Enfin

$$
x_{1}(q)=\sqrt{\frac{a_{1}\left(a_{2}-a_{3}\right)}{H}} \int_{-a_{1}}^{q} \frac{d q}{2 \sqrt{\left(q+a_{2}\right)\left(q+a_{3}\right)}}
$$

conduit à poser

$$
\sqrt{-q-a_{3}}=\sqrt{a_{2}-a_{3}} \operatorname{ch} \theta, \sqrt{-q-a_{2}}=\sqrt{a_{2}-a_{3}} \operatorname{sh} \theta
$$

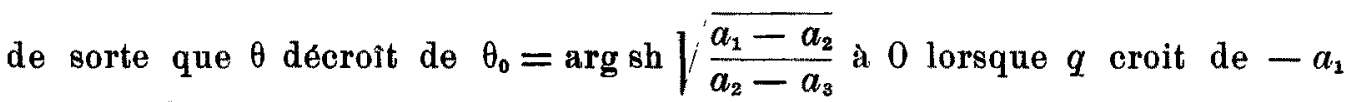
a $-a_{2}$; il vient alors

$$
x_{1}(q)=\sqrt{\frac{a_{1}\left(a_{2}-a_{3}\right)}{H}}\left(\theta_{0}-\theta\right)
$$

en fonction de $\varphi, \theta=\arg \operatorname{sh}\left(\sqrt{\frac{a_{1}-a_{2}}{a_{2}-a_{3}}} \cos \varphi\right)$. En résamé, l'arc $\widehat{M_{0} M_{1}}$ est l'are décrit par le point

$$
\left\{\begin{array}{l}
x_{1}=\sqrt{\frac{a_{1}\left(a_{2}-a_{3}\right)}{H}}\left[\theta_{0}-\arg \operatorname{sh}\left(\sqrt{\frac{a_{1}-a_{2}}{a_{2}-a_{3}}} \cos \varphi\right)\right], \\
x_{2}=\sqrt{\frac{a_{2}\left(a_{1}-a_{3}\right)}{H}} \operatorname{Arcsin}\left(\sqrt{\left.\frac{a_{1}-a_{2}}{a_{1}-a_{3}} \sin \varphi\right),}\right. \\
x_{3}=\sqrt{\frac{-a_{3}\left(a_{1}-a_{2}\right)}{H}} \varphi, \quad 0 \leq \varphi \leq \frac{\pi}{2} .
\end{array}\right.
$$

Les coordonnées de $M_{1}$ sont

$$
\left\{\begin{array}{l}
x_{1,1}=\sqrt{\frac{a_{1}\left(a_{2}-a_{3}\right)}{H}} \theta_{0}, \quad x_{2,1}=\sqrt{\frac{a_{2}\left(a_{1}-a_{3}\right)}{H}} \operatorname{Arcsin} \sqrt{\frac{a_{1}-a_{2}}{a_{1}-a_{3}}}, \\
x_{3,1}=\frac{\pi}{2} \sqrt{\frac{-a_{3}\left(a_{1}-a_{2}\right)}{H}}
\end{array}\right.
$$


Le long de l'arc suivant $\widehat{M_{1} M_{2}}$, le point associé $m$ de $\gamma$ a les cordonnées $\left(24^{\prime}\right)$ et $q$ décroit de $-a_{2}$ à $-a_{1}$, done les coordonnées $x_{i}(q)$ du point courant $M$ sont

$$
\bar{x}_{i}(q)=x_{i, 1}+\int_{-a_{2}}^{q} \bar{\xi}_{i}(q) \frac{d s}{d q} d q=x_{i, 1}-\int_{-a_{2}}^{q} \bar{\xi}_{i}(q)\left|\frac{d s}{d q}\right| d q .
$$

Il vient ainsi

$$
\left\{\begin{array}{l}
x_{1}=x_{1,1}-\int_{-a_{u}}^{q} \xi_{1}(q)\left|\frac{d s}{d q}\right| d q=2 x_{1,1}-x_{1}=\sqrt{\frac{a_{1}\left(a_{2}-a_{3}\right)}{H}\left(\theta_{0}+\theta\right),} \\
\bar{x}_{2}=x_{2,1}+\int_{-a_{2}}^{q} \xi_{2}(q)\left|\frac{d s}{d q}\right| d q=x_{2}, \\
\bar{x}_{3}=x_{3,1}-\int_{-a_{2}}^{q} \xi_{3}(q)\left|\frac{d s}{d q}\right| d q=2 x_{3,1}-x_{3}=\sqrt{\left.\frac{-\frac{a_{3}\left(a_{1}-a_{2}\right)}{H}}{H}-\varphi\right) .}
\end{array}\right.
$$

L'extrémité $M_{2}\left(q=-a_{1}\right.$, donc $\left.\varphi=0\right)$ a les cordonnées

$$
x_{1,2}=2 x_{1,1}, \quad x_{2,2}=0, \quad x_{3,2}=2 x_{3,1} .
$$

Le long de l'are $\widehat{M}_{2} M_{3}, q$ croit de nouveau de $-a_{1}$ a $-a_{2}$, avec les coordonnées $\left(24^{\prime \prime}\right)$ du point $m$ de $\gamma$. Les coordonnées $\bar{x}_{i}(q)$ de son point courant $M$ sont done

$$
\bar{x}_{i}(q)=x_{i, 2}+\int_{-u_{1}}^{q} \bar{\xi}_{i}(q)\left|\frac{d s}{d q}\right| d q
$$

soit

$$
\left\{\begin{array}{l}
\overline{\bar{x}}_{1}=x_{1,2}-\int_{-a_{1}}^{q} \xi_{1}(q)\left|\frac{d s}{d q}\right| d q=x_{1,2}-x_{1}=\bar{x}_{3}, \\
\bar{x}_{2}=x_{3,2}-\int_{-a_{1}}^{q} \xi_{2}(q)\left|\frac{d s}{d q}\right| d q=-x_{2}=-\bar{x}_{2}, \\
\overline{\bar{x}}_{3}=x_{3,2}+\int_{-\alpha_{1}}^{q} \xi_{s}(q)\left|\frac{d s}{d q}\right| d q=2 x_{3,1}+x_{3}=2 x_{3,2}-\bar{x}_{3} .
\end{array}\right.
$$


Les coordonnées de $M_{3}$ sont

$$
x_{1,3}=x_{1,1}, \quad x_{2,3}=-x_{2,4}, \quad x_{3,3}=3 x_{3,1} .
$$

Le long du quatrième arc $M_{3} M_{4}, q$ décroit de $-a_{2} \grave{a}-a_{1}$; le point $m$ associé a les coordonnées $\left(24^{\prime \prime}\right)$, et les coordonnées $\overline{\bar{x}}_{i}(q)$ du point courant $M$ de $C$ sont

$$
\overline{\bar{x}}_{i}(q)=x_{i, 3}-\int_{-a_{2}}^{q} \overline{\bar{\xi}}_{i}(q)\left|\frac{d s}{d q}\right| d q,
$$

soit

$$
\left\{\begin{array}{l}
\bar{\equiv}_{1}=x_{1,3}+\int_{-a_{2}}^{q} \xi_{1}(q)\left|\frac{d s}{d q}\right| d q=x_{1,3}-x_{1,1}+x_{1}=x_{1}, \\
\overline{\overline{x_{2}}}=x_{2,3}-\int_{-\alpha_{2}}^{q} \xi_{2}(q)\left|\frac{d s}{d q}\right| d q=x_{2,3}+x_{2,4}-x_{2}=-x_{2}, \\
\overline{\bar{x}}_{3}=x_{3,3}-\int_{-a_{2}}^{q} \xi_{3}(q)\left|\frac{d s}{d q}\right| d q=2 x_{3,2}-x_{3} .
\end{array}\right.
$$

Les coordonnées de $M_{4}$ sont

$$
x_{1,4}=x_{2,4}=0, \quad x_{3,4}=2 x_{3,2} .
$$

Les expressions $\left(28^{\prime}\right)$ se déduisent de (28) en changeant $\varphi$ en $\pi-\varphi$, ce qui change $\theta$ en $-\theta$, donc l'arc $\widehat{M_{8} M_{2}}$ est représenté par les senles équations (28) où $\varphi$ croit de 0 à $\pi$. On voit également que $\widehat{M_{2} M_{4}}$ est symétrique de $\widehat{M_{0} M_{2}}$ par rapport à la parallèle à $0 x_{1}$ qui passe par $M_{z}$, et qu' on peut le repré. senter par les equations de $\widehat{M_{0} M_{2}}$ après le changement de $\varphi$ en $2 \pi-\varphi$ $(0 \leq \varphi \leq \pi)$. (28) représente donc l'arc $\widehat{M_{0} M_{4}}$ en $y$ faisant varier $\varphi$ de 0 a $2 \pi$.

On peut naturellement prolonger cet arc par la translation de mesure $\pm 2 \pi \sqrt{\frac{-a_{3}\left(a_{1}-a_{2}\right)}{H}}$, parallèlè à $0 x_{3}$, itérée, ce qui revient à faire varier $\varphi$ de $-\infty$ à $+\infty$ pour former une courbe indéfinie $C$. La suppression de la translation parallele à $0 x_{1}$ que représente le terme en $\theta_{0}$ dans (28) et une 
symétrie par rapport à $x_{2} 0 x_{3}$ donne de cette courbe $C$ la réprésentation

$$
\left\{\begin{array}{l}
x_{1}=\sqrt{\frac{a_{1}\left(a_{2}-a_{3}\right)}{H}} \arg \operatorname{sh}\left(\sqrt{\frac{a_{1}-a_{2}}{a_{2}-a_{3}}} \cos \varphi\right), \\
x_{2}=\sqrt{\frac{a_{2}\left(a_{1}-a_{3}\right)}{H}} \operatorname{Arcsin}\left(\sqrt{\frac{a_{1}-a_{2}}{a_{1}-a_{3}}} \sin \varphi\right), \\
x_{3}=\sqrt{\frac{-a_{3}\left(a_{1}-a_{2}\right)}{H}} \varphi, \quad-\infty<\varphi<\infty .
\end{array}\right.
$$

L'analogie avec l'hélice eirculaire est remarquable. Celle-ci, qui est une solution limite de notre problème, s'en déduit d'ailleurs tout de suite lorsque $a_{z}$ tend vers $a_{1}$. Les expressions limites de $(30)$ sont en effet

$$
\left\{\begin{array}{l}
x_{1}=\frac{V \overline{a_{1}}}{a_{1}-a_{3}} \cos \varphi \\
x_{2}=\frac{\sqrt{a_{1}}}{a_{1}-a_{3}} \sin \varphi \\
x_{3}=\frac{\sqrt{-a_{3}}}{a_{1}-a_{3}} \varphi
\end{array}\right.
$$

